rev.relac.int.estrateg.segur.7(2):171-190,2012

\title{
IMPLICACIONES DE LOS ACUERDOS DE PAZ DE 1996 DE GUATEMALA EN LA BALANZA COMERCIAL, 1996-2006*
}

\author{
Sory Vanessa Gómez Ramírez**
}

\section{RESUMEN}

En diciembre de 1996, el Gobierno de Guatemala y la Unidad Revolucionaria Nacional Guatemalteca (URNG), firmaron los Acuerdos de Paz Firme y Duradera poniendo fin a 36 años de conflicto armado en el país. Los Acuerdos de Paz se dividieron en seis temas o acuerdos sustantivos y cinco temas o acuerdos operativos para facilitar el cumplimiento de diversos temas operativos y contraer las causas económicas, sociales y políticas que generaron el conflicto. Pasada una década, se ha podido observar que las causas que originaron el conflicto aún persisten; entre ellas, encontramos que la causa principal es la poca inversión en gasto público social que ha existido durante la historia de Guatemala. Por tal motivo, el propósito del presente ensayo es mencionar la importancia que significa la inversión pública en educación, para el éxito del comercio exterior y la balanza comercial de un Estado.

Palabras Clave: Acuerdos de Paz de Guatemala, temas sustantivos, inversión social, educación, comercio exterior

* Este artículo es producto de una investigación que se inicio en el pregrado para optar por el título de profesional en relaciones internacionales y estudios políticos de la UMNG y se finalizó en el desarrollo de la maestría en relaciones y negocios internacionales, de la misma universidad. La investigación completa se puede consultar en la biblioteca de la UMNG.

** Magister en Relaciones y negocios internacionales de la universidad Militar Nueva Granada, Bogotá, Colombia.

Correo electrónico: sory9388@hotmail.com 


\title{
1996 GUATEMALA'S PEACE AGREEMENT AND THEIR IMPLICATIONS FOR A COMMERCIAL BALANCE 1996-2006
}

\begin{abstract}
In 1996, the Guatemalan Government and the guerrilla group Guatemalan National Revolutionary Unit "URNG", signed the so-called Firm and Lasting Peace Agreement, ending 36 years of armed conflict in the country. The Peace Agreement is composed of six fundamental issues and five operative issues, in order to facilitate its compliance and to diminish the economic, social and political causes that originated the conflict. However, a decade later, we find that the reasons for the conflict still exist; among them, the main cause is the insufficient investment in social matters that has existed in Guatemalan history. For that reason, the purpose of this essay is to stress the importance of public investment in education, for the success of foreign trade and the commercial balance of a State.
\end{abstract}

Key words: Guatemalan Peace Agreement, substantive issues, social investment, education, foreign trade.

\section{IMPLICAÇÕES DOS ACORDOS DE PAZ DE 1996 DA GUATEMALA NA BALANÇA COMERCIAL DE 1996 A 2006}

\section{RESUMO}

Em 1996, o Governo da Guatemala e a Unidade Revolucionária Nacional Guatemalteca (URNG) assinaram os Acordos de Paz Firme e Duradoura terminando com 36 anos de conflito armado no país. Os Acordos de Paz foram divididos em seis temas ou acordos substantivos e cinco tópicos ou acordos operacionais (Pérez Molina, 1999, p1) para facilitar seu cumprimento e reduzir as causas econômicas, sociais e políticas que geraram o conflito. Depois de uma década, tornou-se evidente que as causas que originaram o conflito ainda existem, entre elas, descobrimos que a principal causa é a falta de investimento na despesa pública, que tem ocorrido ao longo da história da Guatemala. Portanto, o objetivo deste ensaio é o de mencionar a importância que representa o investimento público na educação para o sucesso do comércio exterior e da balança comercial do Estado.

Palavras-chave: Acordos de Paz da Guatemala, questões substantivas, investimento social, educação, comércio exterior. 


\section{INTRODUCCIÓN}

En el presente trabajo hablaremos de los Acuerdos de Paz de Guatemala de 1996, que representan el fin de treinta y seis años de conflicto armado interno; son considerados el marco político para tratar de resolver los problemas de fondo del país, y a través de ellos tratar de darle un contenido sustantivo a la paz; debe tenerse en cuenta que para vivir en un ambiente de paz no basta con que haya ausencia de la guerra; también es necesario resolver las causas que generan los conflictos. En ese orden de ideas, en la primera parte se mencionará brevemente los antecedentes de los Acuerdos de Paz y las etapas del proceso de paz; los instrumentos jurídicos y acuerdos firmados de 1986 a 1996; y las disposiciones que se plasmaron en el acuerdo, para hacer efectivo el cumplimiento de los temas sustantivos. En la segunda parte, se observará la balanza comercial de Guatemala durante los años de 1996 al 2006, junto a los más importantes indicadores sociales; fue en la época anterior al conflicto armado, que la cobertura y acceso a la educación, al igual que a los servicios públicos, de salud, así como la falta de oportunidades de empleo y altos índices de pobreza y violencia, las que generaron algunos de los problemas socioeconómicos que dieron origen al mismo. Hay que resaltar que en la era de la globalización económica en que vivimos, la educación es generadora de conocimiento y capital humano, lo cual es indispensable para que haya crecimiento económico y desarrollo social.

\section{ANTECEDENTE DE LOS ACUERDOS DE PAZ FIRME Y DURADERA DE 1996}

En primera instancia, es importante mencionar el origen político del conflicto armado guatemalteco, antes de hablar de los Acuerdos de Paz, suscritos entre el Gobierno de Guatemala y la guerrilla Unidad Revolucionaria Nacional Guatemalteca (URNG), en el año de 1996. Hay que resaltar que el origen político del conflicto se dio dentro del marco de la Guerra Fría; y se remonta al año 1954, cuando el Coronel Carlos Castillo Armas, con el apoyo de la extrema derecha de Guatemala y del Gobierno de los Estados Unidos, a través de la Agencia Central de Inteligencia (CIA) invadieron Guatemala, desde el vecino país de Honduras y derrocaron al Presidente de turno, Jacobo Árbenz Guzmán ${ }^{1}$ (Cullather, 2004). Ese mismo año, el Coronel Castillo Armas fue electo Presidente de la República e inició un período de gobiernos liberales anticomunistas, que siguió la Política de Seguridad Nacional de los Estados Unidos (de lucha y contención del comunismo); y cerraron los espacios políticos de participación democrática a los partidos de izquierda, dando paso a una crisis socio-política en Guatemala.

1. En 1952, el gobierno de los Estados Unidos presentó a Jacobo Árbenz como supuesto comunista, por lo que la Agencia Central de Inteligencia (CIA), organizó la operación PBSUCCESS, entrenó y financió un ejército (Movimiento de Liberación Nacional) para darle un Golpe de Estado. Se llevó a cabo un bombardeo masivo a la región central de Guatemala, con aviones cazabombarderos P-51 de EE.UU (Cullather, 2004). 
Todo ello creó malestar en la población guatemalteca y fue una de las razones por las cuales surgieron movimientos populares y organizaciones subversivas. Así, "el movimiento guerrillero tuvo entre sus principales fundadores a ex oficiales del Ejército de Guatemala." (Pérez, 1998, p. 1). En 1963, cuando un grupo de oficiales ${ }^{2}$-entrenados como Rangers en Fort Brag, Estados Unidos-, intentaron derrocar al General Miguel Idígoras Fuentes por la forma autocrática de gobernar, una vez fracasaron, terminaron por huir a las montañas y con el tiempo establecieron lazos con Cuba. Posteriormente, se convirtieron en el núcleo de las Fuerzas Armadas Rebeldes (FAR); y para el año de 1982, cuatro de los grupos guerrilleros insurgentes, formaron la Unidad Revolucionaria Nacional Guatemalteca, entre los que estaban: el Partido Guatemalteco del Trabajo PGT; la Organización del Pueblo en Armas ORPA; las Fuerzas Armadas Rebeldes FAR; y el Ejército Guerrillero de los Pobres EGP (Aragón, 2000, p. 6).

Fue hasta el año de 1986 cuando el ambiente político nacional empezó a cambiar al ser electo Presidente de La República el Licenciado Marco Vinicio Cerezo Arévalo ${ }^{3}$, que marcó el inicio de gobiernos civiles en el poder. A nivel regional, a través de las Reuniones de Esquipulas $I^{4}$ de 1986 y Esquipulas II de 1987, comenzó un proceso democratizador y pacificador en la región centroamericana. Puesto que el objetivo de Esquipulas I fue poner fin a los conflictos armados internos en Guatemala, El Salvador y Nicaragua, y el de Esquipulas II establecer las bases para la paz y la democracia en Centroamérica, con ello los "Presidentes Centroamericanos se comprometieron a: iniciar un diálogo político en cada país para impulsar la democracia; otorgar amnistía a los insurgentes; establecer una Comisión Nacional de Reconciliación; y exhortar el cese de hostilidades" (Rodríguez 2006, p. 1). A pesar que el Presidente Cerezo fue el gestor de las iniciativas de Esquipulas I y II, durante su Gobierno no fue posible que la guerrilla guatemalteca acudiera a la mesa de negociación con el gobierno.

Fue hasta el año de 1991 que ambas partes discutieron la agenda de negociación, que logró concretarse al dividir los temas en operativos y sustantivos. Los temas operativos son los

2. El descontento popular y el rechazo a la corrupción, hizo que en 1960 surgiera el Movimiento 13 de Noviembre con el Capitán Alejandro de León Aragón, el Coronel Loarca, el Teniente Marco Antonio Yon Sosa, los Subtenientes Luis Trejo Esquivel y Luis Augusto Turcios Lima. El movimiento empezó siendo una rebelión militar en el Fuerte Matamoros; pero, al fracasar, sus líderes se trasladaron a la Sierra de las Minas y continuaron su lucha por la vía de las armas. Al principio tenían una perspectiva nacionalista/reformista, que deseaba restituir la credibilidad al sistema político, borrar la corrupción, depurar su institución y modernizarla; pero después se convirtió en un programa socialista que reclamó tierras para los campesinos. (Wagner, 2005).

3. Licenciado Vinicio Cerezo Arévalo, en 1986 fue primer presidente civil electo democráticamente, después de 32 años de gobiernos militares en el poder. En esa época era el máximo dirigente del Partido Democracia Cristiana Guatemalteca.

4. La iniciativa de Esquipulas I en 1986 fue firmada por los presidentes centroamericanos: Vinicio Cerezo Arévalo de Guatemala, Napoleón Duarte de El Salvador, José Azcona de Honduras, Daniel Ortega de Nicaragua y Oscar Arias de Costa Rica. 
relacionados con la desactivación del enfrentamiento armado e incorporación de los grupos alzados en armas a la vida civil; y los temas sustantivos son los relacionados con la problemática de fondo del país. El período que abarca las negociaciones de paz hasta la firma de los Acuerdos de 1996, el General Otto Pérez Molina (1998), lo divide en tres etapas: la primera, que duró aproximadamente dos años, el moderador fue el presidente de la Comisión Nacional de Reconciliación y la Organización de Naciones Unidas participó como observador. Durante esta etapa, los avances sobre el tema de derechos humanos fueron muy escasos, debido a que la posición de ambas comisiones fue muy dura, hubo resistencia y desconfianza entre las partes. En la segunda etapa, se cambio el esquema anterior; la Organización de las Naciones Unidas pasó de observador a moderador del proceso de paz; se estableció un Grupo de Países Amigos; se amplió la participación civil por medio de la Asamblea de la Sociedad Civil; y se fijó un nuevo calendario. Al intervenir las Naciones Unidas como moderadora, se estableció una Misión para la Verificación de los Derechos Humanos en Guatemala (MINUGUA). Durante esta etapa, varios oficiales de la comisión y representantes de la URNG se reunieron de manera informal cuando negociaban temas delicados y difíciles de llegar a un acuerdo y para entender el punto de vista de sus oponentes. La tercera etapa se caracterizó por un clima de mayor confianza entre las partes y el número de miembros de las comisiones se redujo de diez a cuatro miembros, lo que hizo que se agilizaran las discusiones. Durante este proceso, fue clave para el Ejército de Guatemala y crucial para el éxito del proceso de paz, que tuvieran: un representante en la Comisión de Paz; un contacto directo entre la mesa de negociación y diálogo interno entre sus miembros; también que los temas de Seguridad y Defensa Nacional estuvieron a cargo de la Comisión de Apoyo conformada por un grupo de oficiales que analizaron los temas, hicieron observaciones, recomendaciones y transmitieron la información a los miembros de la institución.

Se debe resaltar que en 1996 se logró la firma de Los Acuerdos de Paz debido al cambio que se dio en el curso de las negociaciones de paz, con la nueva conducción política, la cual "orientó su esfuerzo negociador hacia la conclusión del enfrentamiento armado interno mediante la estrategia de ampliar el espacio político de la insurgencia, producto de una concertación política que superó el fondo y la forma de la negociación y que, al otorgar a los insurgentes la beligerancia que anteriormente no tenían, estimuló el proceso de su reinserción política" (Rosada, 1998, p. 18). En segundo lugar, por haber dividido los temas de la Agenda de Negociación en sustantivos y operativos; y en tercer lugar, el Ejército de Guatemala, a pesar que en un principio una parte del mismo se opuso al proceso de negociación, con el tiempo fue la misma institución la que más apoyo brindó, porque consideró que el proceso de paz era la mejor manera de terminar con el conflicto armado interno.

A continuación se presenta el cuadro de las tres fases del proceso de paz, en el cual participaron actores internacionales como el Grupo de Contadora y Organización de Las Naciones Unidas; y actores nacionales de cada Estado centroamericano. 
Tabla 1. Etapas del Proceso de Paz.

\begin{tabular}{|c|c|c|c|}
\hline & Escenarios & Actores relevantes & Consenso logrados \\
\hline Fase 1 & $\begin{array}{l}\text { Iniciativas } \\
\text { regionales } \\
\text { (Esquipulas } \\
\text { I y II) }\end{array}$ & $\begin{array}{l}\text { Mandatarios centroame- } \\
\text { ricanos; DC; Ejército; Comi- } \\
\text { sión Nacional de Reconci- } \\
\text { liación (CNR); Conferencia } \\
\text { Episcopal (CEG); comuni- } \\
\text { dad internacional y Grupo } \\
\text { de Contadora. }\end{array}$ & $\begin{array}{l}\text { - Procedimiento para establecer la paz firme y dura- } \\
\text { - Acta (Acuerdo de Esquipulas II) } \\
\text { Verificación y Seguimiento. } \\
\text { - Acuerdo de creación de la CNR. }\end{array}$ \\
\hline Fase 2 & $\begin{array}{l}\text { Proceso } \\
\text { de Oslo }\end{array}$ & $\begin{array}{l}\text { URNG (Comisión Político- } \\
\text { diplomática); Gobierno } \\
\text { (CNR); Sector Institucional } \\
\text { del Ejército; CEG. }\end{array}$ & $\begin{array}{l}\text { - Acuerdo básico para la búsqueda de la paz por me- } \\
\text { dios políticos (Acuerdo de Oslo). } \\
\text { - Acuerdo de procedimiento para la búsqueda de la } \\
\text { paz por medios políticos (Acuerdo de México). } \\
\text { - Acuerdo Marco sobre democratización para la bús- } \\
\text { queda de la paz por medios políticos. }\end{array}$ \\
\hline Fase 3 & $\begin{array}{l}\text { Mediación } \\
\text { de NU }\end{array}$ & $\begin{array}{l}\text { URNG; Gobierno/Ejército; } \\
\text { Asamblea Sociedad Civil } \\
\text { ASC; Naciones Unidas NU. }\end{array}$ & 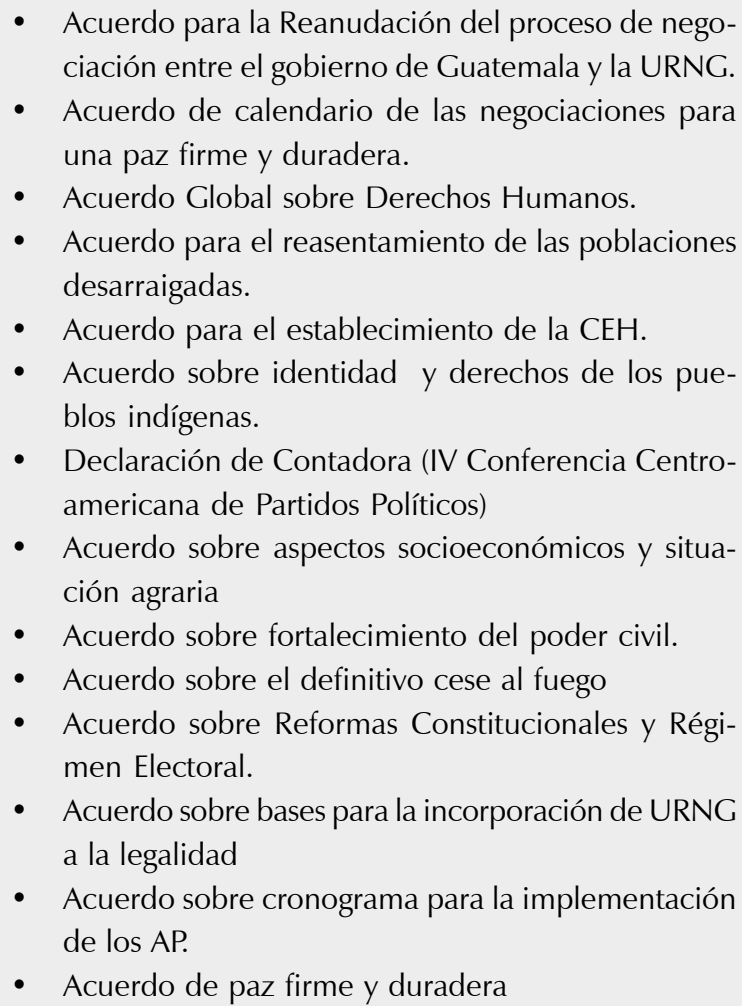 \\
\hline
\end{tabular}

Fuente: Aragón (2000) 


\subsection{INSTRUMENTOS JURÍDICOS Y ACUERDOS FIRMADOS DE 1986 A 1996}

En el año de 1986, los instrumentos que se incorporaron en el ordenamiento jurídico nacional, con el propósito de poner fin al conflicto armado interno fueron: la ley de amparo y habeas corpus. En el ámbito internacional, se firmó el Acuerdo de "Esquipulas I, con los Presidentes Centroamericanos para pacificar y democratizar la región" (Fingermann, 2007, p. 1). En el año de 1987, se firmó el "Acuerdo de Esquipulas II, y con ello, se creó en el país la Oficina del Procurador de los Derechos Humanos y la Comisión Nacional de Reconciliación, con el objetivo de propiciar el diálogo entre el Gobierno y la URNG; como también permitir mayor participación política" (Fingermann, 2007, p. 2). En 1989 se firmó el Acuerdo de Oslo, para buscar una salida negociada al conflicto.

En la década de 1990 se firmaron los siguientes acuerdos: el Acuerdo sobre Derechos Humanos; el Acuerdo para el Restablecimiento de las Personas Desplazadas por el Conflicto Armado; y el Acuerdo sobre el Esclarecimiento Histórico (1994); el Acuerdo sobre Identidad y Derecho de los Pueblos Indígenas (1995). Y finalmente el "Acuerdo sobre Aspectos Socioeconómicos y Situación Agraria, el Acuerdo de Fortalecimiento del Poder Civil y Función del Ejército en una Sociedad Democrática en 1996"5 (Fingermann, 2007, p. 2). Los acuerdos sobre aspectos socioeconómicos y situación agraria, se firmaron al final, por ser los temas más sensibles y fundamentales para la reforma estructural del Estado. Todos los acuerdos antes mencionados son parte del Artículo 15 de Los Acuerdos de Paz Firme y Duradera de Guatemala de 1996.

\subsection{LOS ACUERDOS DE PAZ DE 1996 Y EL CUMPLIMIENTO DE LOS TEMAS SUSTANTIVOS Y OPERATIVOS, 1996-2006}

En la Ciudad de Querétaro, México, el 25 de julio de 1991, se suscribió el Temario General de México, que dividió la agenda de negociación en temas sustantivos y operativos; ello se hizo con el fin de acordar un procedimiento para la búsqueda de la paz por medios políticos; se estableció como primer punto: que la identidad y pluriculturalidad de la población guatemalteca, serviría de base fundamental para tratar posteriormente los problemas que dieron origen al conflicto armado interno. En 1996, finalizó la etapa de negociación y se firmaron los Acuerdos de Paz el 29 de diciembre de ese mismo año, durante el Gobierno del Presidente Álvaro Arzú Irigoyen.

5. Los Acuerdos de Paz de 1996 trataron de marcar los cambios en las estructuras del poder y la desmilitarización de la sociedad y del Estado, al proponer la creación de nuevas estructuras de seguridad civil y la reconversión de las estructuras militares para garantizar la paz y la democracia. En lo referente a las reformas a la Constitución Política, tratan de modificar: el artículo 244, sobre la integración, organización y funciones del Ejército de Guatemala; el artículo 219, sobre los tribunales militares; hacer un agregado al artículo 186, sobre las funciones del Presidente de la República; el artículo 246, sobre los cargos y atribuciones del Presidente en el Ejército de Guatemala, su doctrina, sistema educativo, recursos y reducción del número de efectivos de 45.000 a 15.500, presupuesto y organismos de inteligencia. 
A nivel regional significó el fin de los procesos de pacificación y democratización de Centroamérica, que inició una década atrás con la iniciativa de Esquipulas I y II en Guatemala; y a nivel nacional, significó "el fin a treinta y seis años de conflicto armado interno en el país; el cual, aproximadamente dejó 200.000 víctimas" (Arciniegas, 2008, p. 1) entre muertos, desaparecidos, desplazados y refugiados. Es importante mencionar que los Acuerdos son el "marco para resolver los problemas nacionales y darle un contenido sustantivo a la paz, considerando que la paz, no sólo es la ausencia de guerra, sino también es resolver las causas que originaron el conflicto" (Asturias 2006, p. 1). Por ello, lo que se pretende es disminuir las causas que lo originaron y transformar al Estado de Guatemala en un "Estado incluyente, pluricultural, multiétnico, respetuoso de los derechos humanos, más justo, más equitativo y con verdadero espíritu democrático" (Asturias, 2006, p. 1).

Hay que resaltar que para darle un contenido sustantivo a la paz, sería más apropiado verlo desde la óptica de un Estado Social de Derecho, y no desde un Estado de Derecho, para garantizar mayor inversión en el gasto público social como también para que el modelo económico sea mixto; ello quiere decir que debe incluir políticas neoliberales y políticas sociales para reducir los índices negativos en el desarrollo humano que puedan generar políticas solo capitalistas.

Los seis temas sustantivos de los Acuerdos de Paz de 1996, que se pretenden cumplir son:

- $\quad$ Fortalecimiento del poder civil y función del ejército en una sociedad democrática.

- Identidad y derechos de los pueblos indígenas.

- Reformas constitucionales y régimen electoral.

- $\quad$ Aspectos socio-económicos.

- Situación agraria.

- $\quad$ Reasentamiento de las poblaciones desarraigadas por el enfrentamiento armado.

\subsection{DISPOSICIONES ESTABLECIDAS EN EL ACUERDO DE PAZ FIRME Y DURADERA, FIRMADO EL 29 DE DICIEMBRE DE 1996}

- El derecho, por cuestiones políticas, de los desarraigados de vivir libremente en Guatemala (Art.3)

- El derecho del pueblo guatemalteco a conocer la verdad de lo acaecido durante las sangrientas luchas internas (Art. 4)

- $\quad$ El derecho de identidad de los aborígenes (Art. 5) 
- $\quad$ El desarrollo económico con justicia social, (Art. 6)

- $\quad$ La inversión prioritaria del gasto público en la solución de los problemas sociales. (Art. 7)

- $\quad$ El deber del Estado en la satisfacción de las necesidades básicas de toda la población. (Art. 8)

- El fortalecimiento del poder civil y participación del Ejército en un ámbito democrático, reorganizando las instituciones. (Art. 10)

- $\quad$ La incorporación de la URNG, a la vida política. (Art. 11)

- $\quad$ El perfeccionamiento del sistema electoral. (Art. 13)

- $\quad$ La anexión de todos los acuerdos suscritos anteriormente a 1996 (Art. 15).

En Los Acuerdos de Paz de 1996, también se incluyó un reconocimiento especial a todos los organismos internacionales, especialmente la mediación de las Naciones Unidas en el proceso de paz y al Grupo de Países Amigos, entre ellos: la República de Colombia, República de Venezuela, el Reino de Noruega, el Reino de España, México y Estados Unidos; puesto que, gracias a la mediación, colaboración y participación de cada uno de los actores internacionales, se pudo hacer realidad el proceso de paz de Guatemala.

Entre los cambios que se han hecho para dar cumplimiento a los temas operativos y sustantivos de Los Acuerdos de Paz de 1996, se pueden mencionar:

- En 1997, el Gobierno, diseñó la estructura institucional para el manejo de los "dineros de la paz"6

- $\quad$ En 1998, se redujo el 33\% del presupuesto del ejército, se redujo el número de efectivos de 46.000 a 30.000 y se cerraron cinco bases militares (Fingermann, 2007)

- $\quad$ En 1999, se "terminó la última fase de repatriación voluntaria, logrando repatriar a 43 mil desplazados." (Pérez, 1998, p. 3)

- $\quad$ En el año 2000, se creó la Academia de la Policía Nacional Civil (PNC), y se llevó a cabo la consulta popular para la reforma a la Constitución Política de la República de Guatemala; pero, la población dijo no a la reforma a la Constitución Política. También, se estableció el calendario y cronograma de implementación y verificación de los Acuerdos de Paz.

6. Los dineros de la paz fueron fondos que aportó la comunidad internacional y el Gobierno de Guatemala, para la implementación de los temas sustantivos y operativos del Acuerdo de Paz. 
- $\quad$ En el año 2001, se aprobó la Ley para la creación de la Dirección de Inteligencia Civil (DIGICl), del Ministerio de Gobernación.

- En el año 2004, por segunda vez se redujo el número de miembros del Ejército de Guatemala, de 30.000 a 15.500 efectivos. También, se creó la Ley Marco para el cumplimiento de los Acuerdos de Paz, decretada por el Congreso de la República de Guatemala ${ }^{7}$, en ejercicio de las atribuciones que le confiere el artículo 171 literal a) de la Constitución Política de la República.

- $\quad$ En el año 2006, se disolvió el Estado Mayor Presidencial (EMP) y en su lugar se creó la SAAS, encargada de la seguridad ciudadana, formada por tres mil ex miembros del Ejército de Guatemala.

Tabla 2. Balance de los Acuerdos de Paz

\begin{tabular}{|c|c|}
\hline Aspecto & Descripción de la situación actual y logros de 1996 al 2006 \\
\hline $\begin{array}{l}\text { Política } \\
\text { Monetaria }\end{array}$ & $\begin{array}{l}\text { - Principal objetivo estabilidad del nivel general de precios bajo una visión } \\
\text { de corto plazo y no integral pues no se articula con la economía real para } \\
\text { crear inversión y producción. } \\
\text { - Continuación de los programas de estabilización y ajuste estructural sus- } \\
\text { tentado en la apertura del mercado externo y la liberalización y moderni- } \\
\text { zación del sistema financiero. } \\
\text { - Privilegia al Estado. } \\
\text { - Crisis financieras, esquema especulativo y corrupto. }\end{array}$ \\
\hline Política Fiscal & $\begin{array}{l}\text { - Debilidad en los ingresos del Estado, los Acuerdos de Paz proyectaban un } \\
\text { aumento del } 4 \% \text { en la carga tributaria, entre } 1995 \text { y 2002, pero fue tan } \\
\text { solo del } 1.5 \% \text {. } \\
\text { - Iniciativas unilaterales del Gobierno. } \\
\text { - Fracaso de la implementación del pacto fiscal y por ende la comisión de } \\
\text { seguimiento del mismo. } \\
\text { - Déficit fiscal. } \\
\text { - Asignación ineficiente de los recursos, no hay reactivación económica y } \\
\text { - Pocial integrada. } \\
\text { - Voca transparencia en la administración tributaria. }\end{array}$ \\
\hline
\end{tabular}

7. Congreso de la República de Guatemala. "Ley Marco para el cumplimiento de los Acuerdos de Paz." Decreto Numero 52-2005. 


\begin{tabular}{|c|c|}
\hline Aspecto & Descripción de la situación actual y logros de 1996 al 2006 \\
\hline $\begin{array}{l}\text { Salud y } \\
\text { Seguridad } \\
\text { Social }\end{array}$ & $\begin{array}{l}\text { - Los compromisos establecidos en los Acuerdos de Paz no se han cumplido } \\
\text { como se esperaba. } \\
\text { - No se ha reformado de fondo el sistema de salud y hay pocas iniciativas } \\
\text { - No he ha fortalecido la seguridad social de manera que se amplíe conside- } \\
\text { rablemente la cobertura. } \\
\text { - Bajo gasto e inversión pública, especialmente en el sector salud. }\end{array}$ \\
\hline Educación & $\begin{array}{l}\text { - } \text { Bajo gasto e inversión pública. } \\
\text { - } \quad \text { Lo se ha alcanzado la meta de ampliación de cobertura. } \\
\text { logrado algunas metas, y obtenido apoyo de la comunidad internacional. } \\
\text { - Adecuación de contenidos educativos y actualización docente. } \\
\text { - Creación de Consejos de Educación a nivel municipal, departamental y } \\
\text { nacional. }\end{array}$ \\
\hline Vivienda & $\begin{array}{l}\text { - Se cumplió con el presupuesto acordado, pero con ayudas externas, pese } \\
\text { a ello no se ven muchas obras ejecutadas. } \\
\text { - Creación de la política nacional de vivienda. } \\
\text { - Presupuesto para desarraigados y desmovilizados. } \\
\text { - Hasta el año } 2008 \text { se creó el Plan de Ordenamiento Territorial y aún no se } \\
\text { ha ejecutado. }\end{array}$ \\
\hline $\begin{array}{l}\text { Política } \\
\text { Laboral }\end{array}$ & $\begin{array}{l}\text { - Se realizaron reformas en la legislación laboral, pero aún faltan beneficios } \\
\text { a favor de los empleados y no de las empresas como tal. } \\
\text { - En el } 2002 \text { aún estaba prohibida la huelga de los trabajadores agrícolas en } \\
\text { tiempos de cosecha. } \\
\text { - No hay acuerdo entre patronos, trabajadores y Estado en materia de salarios. }\end{array}$ \\
\hline $\begin{array}{l}\text { Política hacia } \\
\text { la mujer }\end{array}$ & $\begin{array}{l}\text { - Muchos de los compromisos establecidos en los Acuerdos de Paz, el Go- } \\
\text { bierno los descargó, en el Foro de la Mujer. } \\
\text { Presupuesto reducido para la defensoría de la mujer indígena. Se creó el } \\
\text { plan de equidad y oportunidades 2001-2006, aunque faltan avances en la } \\
\text { materialización del mismo. }\end{array}$ \\
\hline $\begin{array}{l}\text { Desarrollo } \\
\text { Rural y tierra }\end{array}$ & $\begin{array}{l}\text { - Manifiesta el mayor grado de incumplimiento. } \\
\text { - La política del mercado de tierras no modificó la estructura de tenencia de } \\
\text { tierra. }\end{array}$ \\
\hline
\end{tabular}

Fuente: Foro Ecuménico por la Paz y la Reconciliación (2006) 
Conforme el cuadro anterior, se puede observar que el gasto público social en educación continúa siendo bajo y que no se ha alcanzado la meta de ampliar la cobertura educativa. Ello pueda ser porque no se han implementado las disposiciones establecidas en los Acuerdos de Paz, como: el deber del Estado en la satisfacción de las necesidades básicas de toda la población; la inversión prioritaria del gasto público en la solución de los problemas sociales (en el cual, encontramos la educación); y el desarrollo económico con justicia social. Para implementar lo anterior, sería necesario, en primer lugar, el liderazgo y voluntad política de un gobernante, y que se observe lo planteado desde la óptica de un Estado Social de Derecho ${ }^{8}$ y no desde el marco constitucional del Estado de Derecho, porque no garantiza que el Estado satisfaga las necesidades básicas insatisfechas de sus habitantes; como tampoco garantiza la distribución justa de la riqueza con justicia social, ni la prioridad de los problemas sociales en el gasto público; principios que se encuentran en el marco constitucional del Estado Social de Derecho (del Águila, 2000).

\section{BALANZA COMERCIAL DE GUATEMALA, 1996-2006}

El comercio exterior, desde el Siglo XVIII, ha sido de vital importancia para los Estados desarrollados, desde que Adam Smith resaltó que la causa de la riqueza de las naciones se produce cuando cada nación se enfoca "en vender la mayor cantidad de productos al resto del mundo, para acumular la mayor cantidad de oro (riqueza), para su bienestar." (Gudiel y Quinto, 2007, p. 2). Si bien es cierto que en la actualidad los Estados enfrentan varios obstáculos para competir en el mercado mundial, como: la inseguridad, mantener estabilidad macroeconómica, infraestructura, y capital humano; en el presente trabajo sólo se hará referencia al último tema, haciendo énfasis en la educación.

Es importante mencionar la balanza comercial de Guatemala debido que en ella se refleja la actividad económica del país en cuanto al comercio exterior; también, porque las exportaciones contribuyen al desarrollo económico y social al generar divisas, oportunidades de empleo y desarrollo humano; al incorporar mujeres y pequeñas comunidades productoras al mercado laboral, transferir conocimientos y generar actividades económicas complementarias. El comercio es tema prioritario debido que genera riqueza y bienestar a los estados y sus habitantes.

Haciendo mención al primer capítulo del presente trabajo, hay que recordar que en el ámbito político de 1986, con las Reuniones de Esquipulas I y II, se inició el proceso de democratización

8. Las teorías del Estado Social de Derecho tuvieron acogida después de la Segunda Guerra Mundial. Nació en Europa como una forma de organización estatal encaminada a consensuar, realizar justicia social y dignidad humana, mediante la sujeción de las autoridades públicas a los principios, derechos y deberes sociales de orden constitucional. Une la política económica y social para garantizar la subsistencia de la población. 
y pacificación de Centroamérica; pero, en el ámbito económico también hubo cambios; se "inició un proceso de apertura comercial, por el cual se eliminó el 95\% de las restricciones no arancelarias, tales como cuotas y permisos de exportación, y se redujeron los niveles arancelarios desde un promedio de 60\% a 11.38\% en 1996." (AGEXPORT, 2004, p. 10). A raíz de ello, en los países centroamericanos se han venido liberalizando las economías y diseñando políticas que permitan mayor apertura comercial para competir en esta nueva era de la globalización.

A continuación se observará el comportamiento de la balanza comercial de Guatemala, de 1980 a 2006.

Figura 1. Balanza Comercial de Guatemala, 1980-2006

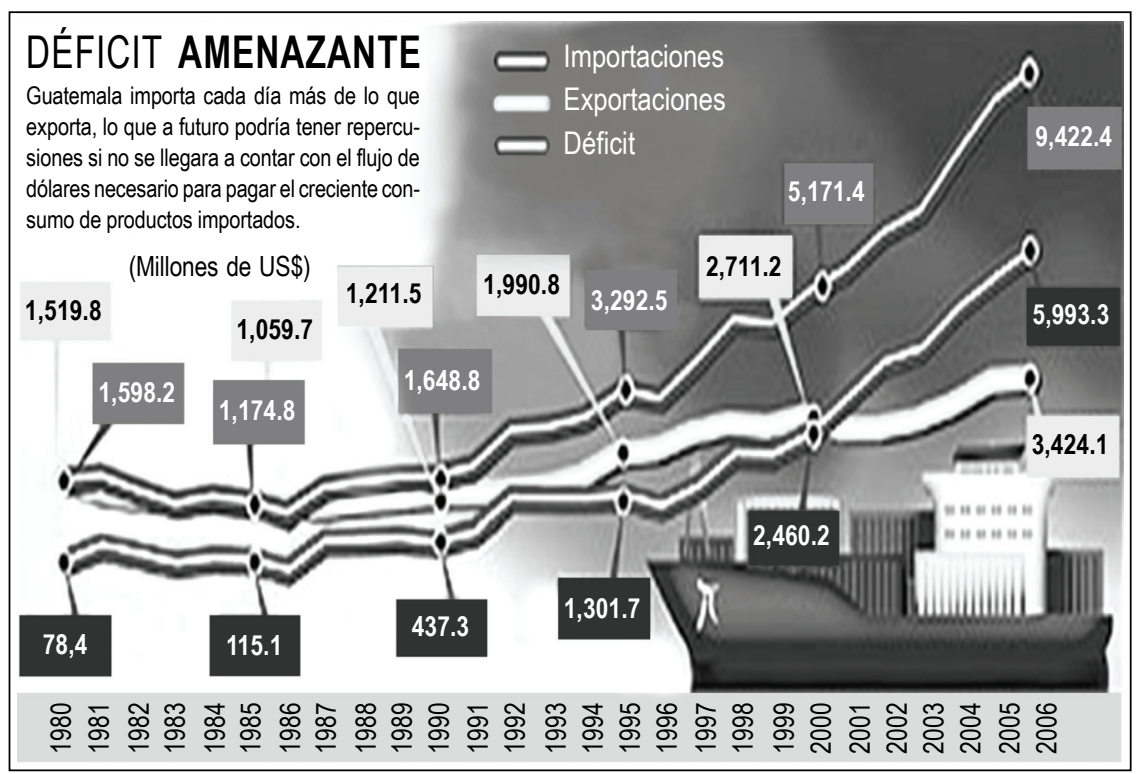

Fuente: Gudiel y Quinto (2007)

En esta tabla se puede observar que en 1980 el déficit comercial fue de 78.4; en el año de 1985, fue de 115.1; en 1990, de 437.3; en el 2000, de 2,460.2; y en el 2006, de 5,998.3. Lo anterior refleja una propensión mayor hacia las importaciones y menor hacia las exportaciones, generando que el déficit comercial aumente año tras año.

Las estadísticas del Banco Central de Guatemala, sólo en el año de 1986, registraron un superávit comercial de US\$84.3 millones, originado por la implementación del Plan de Estabilización que devaluó la moneda, destinado a superar la crisis económica. Conforme el Anuario Estadístico 
2004, de la Comisión Económica para América Latina y el Caribe (CEPAL), a pesar que las exportaciones de Guatemala se han duplicado, de US $\$ 1,519.8$ millones en 1980, a US $\$ 3,424.1$ millones en 2006, las importaciones, se han multiplicado seis veces más de US\$1,598.2 millones en 1980, y a US\$9,422.4 millones en el 2006. Debido a lo anterior, el déficit comercial alcanzó US\$5,998.2 millones, siendo este el segundo más alto de Latinoamérica después de México.

Para Miguel Gutiérrez, analista de Central American Business Intelligence (citado en Gudiel y Quinto, 2007, p. 1), "el déficit comercial del país, crece aceleradamente y no es sostenible a largo plazo, habrá que darse un ajuste en el consumo, tipo de cambio y tasas de interés, porque un dólar barato, motiva el boom de productos importados." Debido a ello, expresa que es necesaria una "ligera devaluación del quetzal, para encarecer las importaciones, motivar las exportaciones y volver a las empresas más competitivas para generar nuevas inversiones, empleos y mayor crecimiento del aparato productivo." Con los ajustes antes mencionados, se podría disminuir el déficit comercial y aumentar el índice de desarrollo humano, utilizando como estrategia de desarrollo las exportaciones. En cuanto al consumo, se puede decir que la cultura de la sociedad guatemalteca refleja gran aceptación por los bienes importados, lo que va en detrimento de los productos nacionales al no permitir que la industria ni el mercado interno nacional crezca y se fortalezca.

Entre los principales productos que se importaron en el año 2008, fueron: materias primas, materiales de construcción, combustibles, bienes de consumo, y bienes de capital; el valor CIF ascendió a US\$7,482.1 millones de dólares (Betancourt, 2008), ello producto del alza petrolera $^{9}$. Para el economista Carlos Barreda (citado en Gudiel y Quinto, 2007, p. 3), uno de los temas "más preocupantes del déficit de la balanza comercial, es la importación de biocombustibles como etanol y biodiesel", porque considera que ambos combustibles podrían producirse en el país, y de esa manera reducir la factura petrolera que en la actualidad genera mayor déficit en la balanza comercial.

Entre los principales productos guatemaltecos de exportación ${ }^{10}$ se pueden mencionar: el café, azúcar, cardamomo, banano y petróleo, que representan el $26.5 \%$ del total de las exportaciones y su valor total fue de US\$3,996.3 millones ${ }^{11}$ (Betancourt, 2008). Es importante mencionar que

9. El valor CIF de los combustibles y lubricantes fue de US\$1,592.8 millones y en lo que respecta a los bienes de consumo, estos representaron el $24.0 \%$ de las importaciones totales.

10. Según datos del Ministerio de Economía, el sector agrícola conformó dos tercios de las exportaciones; el sector pesquero un cuarto del PIB, el sector minero sólo exportó níquel y el sector industrial representó un quinto del PIB. En el sector industrial, las principales industrias son las de alimentos, textiles, madera, cigarrillos, caucho y turismo. El turismo, en la actualidad, es unos de los motores principales de la economía del país, puesto que la industria hotelera reportó más de \$1,000 millones en el 2007, debido al millón de turistas que recibe el país anualmente. 
la mayoría de nuestros productos de exportación son materias primas y no productos con valor agregado o industriales, los cuales generan mayores ingresos. El sector hotelero es el motor principal de la industria por el momento, debido a la cantidad de turistas que recibe el país año con año.

Guatemala también ha firmado Tratados de Libre Comercio (TLC) con Estados Unidos, los países centroamericanos, Taiwán, México y República Dominicana; en el 2008, el total de las "exportaciones fue de US\$3,127.2 millones, las cuales representaron el 78.2\%; y el total de las importaciones, fue de US\$ 4,296.3 millones, representando el 57.5\%." (Betancourt, 2008, p. 15). La balanza comercial reflejó un superávit sólo en las relaciones comerciales con los países centroamericanos y con República Dominicana; y hubo déficit en la balanza comercial con los Estados Unidos, México y Taiwán.

Es importante resaltar que Taiwán logró superar el subdesarrollo viendo hacia el mundo y tomando como base "las exportaciones" como estrategia de crecimiento económico. Para que las exportaciones de Guatemala sean competitivas y que los gobiernos afronten el reto del crecimiento económico sostenible, la competitividad "debe originarse en la fortaleza y la eficiencia de la infraestructura productiva y técnica, así como en los elementos que determinan el entorno económico, social e institucional en que se manifiesta." (AGEXPORT, 2006, p. 5).

Al observar que el Producto Interno Bruto (PIB) per cápita en dólares correspondiente al año 2000 fue de 3900 y el del 2008 fue de 5400, tenemos que presenta un crecimiento económico anual favorable. A continuación, se mencionarán algunos indicadores sociales para observar si ese crecimiento económico se ve reflejado en desarrollo social y mejores condiciones de vida para los habitantes.

\subsection{INDICADORES DE POBREZA, SALUD, EDUCACIÓN Y TRABAJO}

Conforme al cuadro siguiente, se observa que los pueblos indígenas presentan los "peores índices de pobreza extrema, falta de acceso al trabajo, y falta de servicios sociales como educación, salud, justicia e inserción a la vida pública y privada."122 (PNUD, 2006). Los datos anteriores

11. La relación comercial con estos países fue la siguiente: con Estados Unidos, las exportaciones fueron US $\$ 1,646.3$ millones y las importaciones de US\$2,712.4. Con Centroamérica, las exportaciones fueron US $\$ 1,158.0$ millones y las importaciones US $\$ 795.0$ millones. Con México las exportaciones fueron de US\$251.0 millones y las importaciones de US $\$ 720.3$ millones. Con Taiwán las exportaciones fueron de US\$11.3 millones y las importaciones US $\$ 56.3$ millones. Con República Dominicana las exportaciones fueron de US\$60.6 millones y las importaciones US\$12.3 millones.

12. Disponible en http://www.ec.europa.eu. "Desafíos del País para fortalecer la cohesión social.", p. 2. Consultado el 12 de diciembre de 2008. 
Tabla 3. Pueblos Indígenas, Pobreza y Desarrollo Humano en Guatemala: 1994-2006

\begin{tabular}{|c|c|c|}
\hline 1994 al año 2004 & Población indígena & Población ladina \\
\hline $\begin{array}{l}\text { Educación } 15 \text { a } 31 \text { años } \\
\text { Educación } 15 \text { a } 64 \text { años }\end{array}$ & $\begin{array}{l}\text { Años de escolaridad } 3,5 \\
53 \% \text { puede leer y escribir. }\end{array}$ & $\begin{array}{l}\text { Años de escolaridad } 6,3 \\
82 \% \text { puede leer y escribir. }\end{array}$ \\
\hline $\begin{array}{l}\text { Deserción primer año } \\
\text { escolar }\end{array}$ & El $44 \%$ & El $31 \%$ \\
\hline Oportunidad de empleo & Subempleados 15\% & Subempleados $22 \%$ \\
\hline $\begin{array}{l}\text { Salud cobertura } \\
\text { No atención prenatal } \\
\text { Partos en hospitales }\end{array}$ & $\begin{array}{l}\text { El } 5 \% \\
\text { El } 27 \% \\
\text { El } 15 \%\end{array}$ & $\begin{array}{l}\text { El } 18 \% \\
\text { El } 18 \% \\
\text { El } 51 \%\end{array}$ \\
\hline $\begin{array}{l}\text { Conoce algún método anti- } \\
\text { conceptivo } \\
\text { Utiliza algún método anticon- } \\
\text { ceptivo }\end{array}$ & $\begin{array}{l}\text { El } 32 \% \\
\text { El } 12 \%\end{array}$ & $\begin{array}{l}\text { El } 71 \% \\
\text { El } 57 \%\end{array}$ \\
\hline $\begin{array}{l}\text { Acceso a servicios } \\
\text { Energía eléctrica }\end{array}$ & $\begin{array}{l}\text { Área rural } 50 \% \\
\text { Área urbana } 89 \%\end{array}$ & $\begin{array}{l}\text { Área rural } 62 \% \\
\text { Área urbana } 97 \%\end{array}$ \\
\hline
\end{tabular}

Fuente: elaboración propia conforme datos Hall G, Patrinos H. (2006)

podrían ser el resultado del presupuesto limitado en el gasto público social, en temas tan importantes como educación, que son generadores de conocimiento y oportunidades de empleo; de esta manera se genera que un alto porcentaje de la población quede excluida del desarrollo económico y social del país.

El interés sobre el comercio internacional y la balanza comercial radica en que el Estado guatemalteco no ha generando las condiciones necesarias para incentivar las exportaciones, ya que no se ha visto reflejado en mayor riqueza y desarrollo humano para sus habitantes; en el "año 2000 , el $40.5 \%$ de los guatemaltecos eran pobres y el $15.7 \%$ extremadamente pobres" (AGEXPORT, 2006, p. 7), en el año 2004, el índice de pobreza subió al 56.2\%; y el 16\% de la población tuvo ingresos menores de US\$1.00 al día. Conforme el índice de Desarrollo Humano del año 2004, Guatemala descendió al puesto 121; y en el año 2005, descendió al puesto 118 de 177 países. Según estadísticas del Programa de Naciones Unidas para el Desarrollo, ocupó el último lugar de los países centroamericanos, y comparado con el resto de países "latinoamericanos tiene 0.673 de IDH, lo cual lo sitúa en el penúltimo lugar, ocupando el último lugar Haití 
con 0.482 de IDH." (Barreda, 2007, p. 7). Para el año "2006, se situó con un índice medio, ocupando el puesto 96." (Betancourt, 2008).

Para mejorar las condiciones de vida de los habitantes, es necesario "generar oportunidades de trabajo, crédito y asistencia técnica a las micro, pequeñas y medianas empresas; así como una adecuada política fiscal y tributaria, para elevar el nivel educativo de la población y promover el desarrollo rural." (Barreda, 2007, p. 4). En cuanto a la balanza comercial, se puede decir que algunos de los factores que han incidido negativamente son: el alza de los precios del petróleo de los últimos años, la falta de incentivos a las exportaciones, mantener una economía agrícola, la poca inversión social para la formación de capital humano y la cultura de consumo que privilegia los productos importados; esto último puede ser debido a la ausencia de una economía industrial fuerte y competitiva, que sea capaz de suplir las necesidades del mercado interno nacional.

En lo que respecta a la formación de capital humano, a través de la educación, hay que resaltar que para cualquier Estado debe ser de suma importancia, puesto que en la actualidad vivimos en la era de la globalización económica, basada en la información y el conocimiento. Debido a ello, es necesario que los Estados se preocupen por elevar los índices de educación de sus habitantes, para lograr avances positivos que se vean reflejados en crecimiento económico y desarrollo humano. Si bien es cierto que la globalización económica trae consigo crecimiento y desarrollo, esta sólo se produce cuando hay inversión extranjera directa y gasto público social que genere las condiciones necesarias para que las empresas nacionales sean competitivas en el sistema internacional. Ello quiere decir que, aparte de los retos que debe enfrentar el Estado en cuanto a infraestructura, estabilidad macroeconómica y seguridad, es importante aumentar el gasto público social en educación, porque ella es la base para la creación de una buena empresa y el conocimiento es importante para la creación de una buena industria. Michael Porter dice que en este mundo globalizado son las empresas las que compiten y no las personas; pero, a través de las personas, se logra la creación de las empresas.

\section{CONCLUSIONES}

En la primera parte del trabajo se puede observar que en las disposiciones de los Acuerdos de Paz Firme y Duradera de Guatemala de 1996, de los artículos 6, 7 y 8, son principios que hacen parte de un Estado Social de Derecho. Por lo tanto, es pertinente decir, que para lograr el cumplimiento de dichos principios, y hacer los cambios estructurales que necesita el Estado, para solucionar los problemas socioeconómicos que generaron el conflicto armado interno y que en la actualidad aún persisten, sería necesaria la voluntad política de los gobernantes, y que el Estado y la sociedad guatemalteca avancen hacia un Estado Social de Derecho, y evitar que en el futuro los problemas sociales generen descontento popular e inestabilidad política. 
Los esfuerzos del Estado guatemalteco por proporcionar bienestar a sus habitantes han demostrado ser insuficientes; ello se debe a las débiles políticas sociales y económicas, como también por la combinación de factores internos y externos, que han generado desmejoras en las condiciones de vida de sus habitantes. Como los problemas socioeconómicos vividos antes del conflicto armado interno aún persisten y los temas sustantivos de los Acuerdos de Paz no se han cumplido en su mayoría, la comunidad internacional se ha visto en la necesidad de catalogar la situación interna de los habitantes como una situación de pre conflicto, como si en Guatemala se viviese "sin haber superado el post conflicto." (Marroquín, 2001, p. 2).

En cuanto a la segunda parte del trabajo, la importancia de analizar el comercio internacional y tratar de buscar soluciones a los problemas nacionales a través del mismo, se debe a la importancia que representa el comercio en la generación de bienestar a la población y ser motor de crecimiento económico y desarrollo humano, social y político. Por ello, se pudo observar que las implicaciones del cumplimiento de los Acuerdos de Paz de Guatemala, en la balanza comercial durante el período de 1996 al 2006, no ha tenido un impacto significativo; ello se debe a que el modelo de desarrollo económico no ha sido acorde a las necesidades de la población guatemalteca. Lamentablemente el crecimiento económico que se observa en Guatemala no se ha visto reflejado en desarrollo social; los indicadores de pobreza han aumentado, el acceso a la educación todavía es deficiente y falta mucha inversión social para crear capital humano.

Teniendo en cuenta que el tema económico siempre ha estado ligado a todas las relaciones de la sociedad guatemalteca, es importante resaltar que el Estado debe direccionar su política exterior utilizando como estrategia las exportaciones y buscar ventajas competitivas; también, es necesario definir metas claras de crecimiento económico que genere desarrollo social, realizar un plan de desarrollo con inversión social, ampliar la cobertura en educación y salud; reducir los índices de pobreza y desempleo, para incorporar a la mayor cantidad de personas a la actividad económica y productiva del país; también para insertarnos de forma efectiva en la era de la globalización económica, competir en el mercado internacional y nivelar la balanza comercial.

Debido a todo lo anterior, se recomienda continuar avanzando en el cumplimiento de los temas sustantivos de los Acuerdos de Paz de 1996, poniendo especial énfasis en la inversión prioritaria del gasto público en la solución de los problemas sociales; entre ellos, el gasto en educación indispensable para la creación de conocimiento o capital humano, base de la competitividad de las empresas. 


\section{BIBLIOGRAFÍA}

- $\quad$ Aragón González, J. (2000). "Los próximos escenarios para la implementación de los acuerdos de paz de Guatemala." Informe final del concurso: Democracia, derechos sociales y equidad; Estado, política y conflictos sociales. Buenos Aires: CLACSO.

- $\quad$ Arciniegas, A. (2007) "Escepticismo por pocos avances en firma de paz". Diario La Costa. Última consulta: 29 de enero de 2008. Recuperado de: http://www.diariolacosta.com

- $\quad$ Asturias Valenzuela, S. (s.f.) "Balance de los Acuerdos de Paz en Guatemala 1996-2006."

- Banco de Guatemala. (2006). Balanza Comercial de Guatemala (Última consulta: 10 enero 2010). Recuperado de: http://www.banguat.gob.gt/inc/main.asp?id=646\&aud= $1 \&$ lang $=1$

- Barreda, C. (2007) Pobreza, salud y educación en Guatemala. (Última consulta: 10 de diciembre de 2008. Recuperado de: www.albedrío.org.

- $\quad$ Betancourt, J. E. (2008). Comportamiento del Comercio Exterior durante el primer semestre de 2008. Ministerio de Economía, Dirección de análisis económico. Guatemala.

- $\quad$ CIA World Factbook. (s.f.). Guatemala Población. (Última consulta: 12 de agosto 2008). Recuperado de: http://www.indexmundi.com/es/guatemala/población.html.

- $\quad$ Congreso de la República de Guatemala (2008). Ley Marco para el cumplimiento de los Acuerdos de Paz. Decreto Número 52-2005.

- $\quad$ Cullather N. (2004). GUATEMALA. Operación PBSUCCESS. Las operaciones encubiertas de la CIA en apoyo al Golpe de Estado de 1954. Guatemala: Tipografía Nacional de Guatemala.

- Del Águila, R. (2000). "Manual de Ciencia Política". Segunda Edición. Madrid: Editorial Trotta, S.A.

- $\quad$ Fingermann, H. (2007). El Proceso de Paz en Guatemala. (Última consulta: 10 de Enero de 2008). Recuperado de: http://www.laguia2000.com/guatemala/el-proceso-de-paz-enguatemala.

- $\quad$ Foro Ecuménico por la paz y la reconciliación (2002). Acuerdos de paz: situación actual y perspectivas. Guatemala: Foro Ecuménico por la paz y la reconciliación. Guatemala. 
- García Jiménez, C. (2007). Teoría del Estado. (Última consulta: 10 de agosto de 2008). Recuperado de: www.derecho.serviciocolectivo.com.

- Gudiel, V. y Quinto, R. (2007). ¿El Déficit Comercial amenaza a la economía? (Última consulta: 20 de diciembre de 2008. Recuperado de: http://www.elperiódico.com.gt//es/ 20070226/actualidad/37201/.

- Hall, G. y Patrinos H. (2006). Pueblos indígenas, pobreza y desarrollo humano en América Latina: 1994-2004. Banco Mundial en coedición con Mayol Ediciones S.A. Bogotá, Colombia.

- INDEXMUNDI. (s.f.). Producto Interno Bruto de Guatemala. (Última consulta: 5 de enero 2009. Recuperado de: www.indexmundi.com/es/guatemala/producto interno bruto (pib)per_capita.html.

- Instituto Nacional de Estadística de Guatemala INE. (s.f.). "Censos de población 1971, 1983, 1994 y 2002. (Última consulta: 29 de enero 2008). Recuperado de: www.ine.gob.gt.

- Kompass, A. (2007). Representante OACNUDG/GUATEMALA. El Papel de Naciones Unidas en el apoyo al cumplimiento de los Acuerdos de Paz. Parlamento Europeo: Bruselas.

- $\quad$ Pérez Molina, O. (1998). Crisis, Cuál Crisis iEl Proceso de Paz en Guatemala. Guatemala.

- $\quad$ Porter Michael. (1999). La ventaja competitiva de las naciones. Vergara.

- $\quad$ Rodríguez. L. (2006). Celebran 20 años del proceso de paz. En: Periódico Prensa Libre. Guatemala.

- $\quad$ Rosada, H. (1998). El lado oculto de las negociaciones de paz. Transición de la guerra a la paz de Guatemala. Guatemala: Fundación Friederich Ebert Stiftung.

- Unión Europea (2005). Desafíos del País para fortalecer la cohesión social. Borrador para el diálogo. (Última consulta: 12 de diciembre de 2008). Recuperado de: http:// www.delgtm.ec.europa.eu/files/dms/borrador_concept_note.pdf.

- Wagner, R. (2005). Curso de Historia Política de Guatemala II. Guatemala: Universidad Francisco Marroquín. 